\title{
Hemangioma capilar lobular nasal del cornete medio: una causa rara de epistaxis recurrente
}

\author{
Nasal capillary lobular hemangioma of the middle turbinate: \\ a rare cause of recurrent epistaxis \\ José Antonio Marino Silva, ${ }^{*}$ Gerardo Gutiérrez Santos, ${ }^{*}$ Jaime Fernández Espinosa, ${ }^{*}$ \\ Teresa Cristina Cuesta Mejías, ${ }^{\ddagger}$ Manuel Guerrero Hernández ${ }^{\S}$
}

\begin{abstract}
RESUMEN
La epistaxis es la urgencia otorrinolaringológica más común a la que se enfrenta tanto médicos generales como especialistas. La incidencia es incierta, ya que la mayoría se resuelve espontáneamente. Se divide de manera coloquial, en anterior $(85-90 \%)$ y posterior $(15-10 \%)$, y generalmente es la última la que requiere mayor atención médica de urgencia. Las causas son múltiples y pueden ser anatómicas, médicas, tumorales (benignas y malignas) o traumáticas. El clínico debe tener un amplio diagnóstico diferencial para tratar las diferentes causas enfocadas en la etiología. El objetivo del caso clínico es presentar un diagnóstico diferencial en la causa y manejo de epistaxis, al ser una lesión que rara vez se origina en el cornete medio. Presentamos el caso de un paciente masculino de 43 años, previamente sano, quien presentó cuadros recurrentes de epistaxis unilateral. El paciente acudió previamente a nuestra valoración al Servicio de Urgencias en dos ocasiones por esta patología. Requirió en dos ocasiones taponamiento nasal anterior, sin ceder completamente el cuadro. En la revisión endoscópica nasal, se encontró una lesión dependiente del cornete medio derecho de color rojo-azulado, siendo éste el sitio de sangrado. Se realizó tomografía computarizada contrastada de nariz y senos paranasales y resonancia magnética cerebral con gadolinio, encontrándose imagen compatible con lesión vascularizada del cornete medio derecho. Previa angioembolización, se realizó cirugía endoscópica nasal para excisión completa de la lesión con apoyo del neuronavegador. En el estudio histopatológico, se encontró un hemangioma capilar lobular. Se le dio seguimiento al paciente, el cual no presentó recurrencia de la lesión de características benignas.
\end{abstract}

Palabras clave: Epistaxis, hemangioma capilar lobular, angioembolización, neuronavegador.

Nivel de evidencia: IV

\begin{abstract}
Epistaxis is the most common emergency in otolaryngology that general practitioners and specialists confront. Incidence is uncertain because most cases resolve spontaneously. It can be classified in anterior (85-90\%) and posterior (15$10 \%)$ usually and the last is the one that requires more often urgent medical attention. There are multiple causes which can be anatomical, medical, tumoral (benign and malignant) or traumatic. The clinician must have a sound differential diagnosis armamentarium to treat the different causes focused on the etiology. We present the case of a 43 year old male patient, with no previous medical history, with recurrent unilateral epistaxis. The patient was previously seen at the emergency department in two occasions before consulting with us. He had required anterior nasal packing in two occasions without solving the epistaxis. In the endoscopic exploration of the nasal cavity we found a tumor dependent of the middle turbinate with a red-blue appearance, which was the site of bleeding. In the CT scan and MRI of the head we found an image compatible with a vascular tumor dependent of the right middle turbinate. Previous angioembolization, the tumor was excised with nasal endoscopic surgery with the assistance of navigation system. The histopathology was compatible with a lobular capillary hemangioma. The patient was followed up for 6 months with no recurrence. The purpose of this clinical case is to provide another differential diagnosis to the armamentarium of clinicians handling patients with epistaxis, as this tumor rarely appears in the middle turbinate.
\end{abstract}

Keywords: Epistaxis, lobular capillary hemangioma, angioembolization, neuronavigation.

Level of evidence: $I V$
* Otorrinolaringología y Cirugía de Cabeza y Cuello. Miembros de Cuerpo Médico.

₹ Servicio de Patología Clínica.

$\S$ Jefe de Radiología Intervencionista.

Centro Médico ABC Santa Fe. Ciudad de México.

Recibido para publicación: 07/08/2019. Aceptado: 15/11/2019.
Correspondencia: Dr. José Antonio Marino Silva

Av. Carlos Graef Fernández Núm. 154,

Col. Santa Fe, 05300, Cuajimalpa, CDMX. Teléfono: 16647026

E-mail: drmarino88@gmail.com

Este artículo puede ser consultado en versión completa en:

www.medigraphic.com/analesmedicos 


\section{INTRODUCCIÓN}

La epistaxis es la urgencia otorrinolaringológica más común a la que se enfrenta tanto médicos generales como especialistas. La incidencia es incierta, ya que la mayoría se resuelve espontáneamente. Se divide en anterior (85-90\%) y posterior (15-10\%) de manera coloquial, y generalmente la última es la que requiere mayor atención médica de urgencia. Las causas son múltiples y pueden ser anatómicas, médicas, tumorales (benignas y malignas) o traumáticas. ${ }^{1} \mathrm{El}$ clínico debe tener un amplio diagnóstico diferencial para tratar las diferentes causas enfocadas en la etiología.

El hemangioma capilar lobular de la cavidad nasal es un tumor benigno. La presentación usual del tumor es originaria de los tejidos blandos; el sitio más común es el septum y la cabeza anterior del cornete inferior. Existen dos variedades histológicas del hemangioma: una es el hemangioma cavernoso y el otro el capilar. El hemangioma cavernoso está compuesto de endotelio largo cubierto por grandes espacios vasculares. El hemangioma capilar está compuesto por vasos de un tamaño «capilar», cubierto por epitelio aplanado separado por estroma de colágeno. ${ }^{2}$ En imagenología, el hemangioma presenta reforzamiento intenso temprano en la tomografía computarizada contrastada, con bordes definidos, y en la resonancia magnética presenta en $\mathrm{T} 1$ ponderado con contraste, alto reforzamiento de la lesión; asimismo, en T2 refuerza intensamente. ${ }^{3}$ Las imágenes generalmente muestran un aspecto circunscrito localizado, pero en ocasiones puede haber remodelación ósea. En imágenes de radiología intervencionista, muestra vascularidad con múltiples ramificaciones de la lesión.

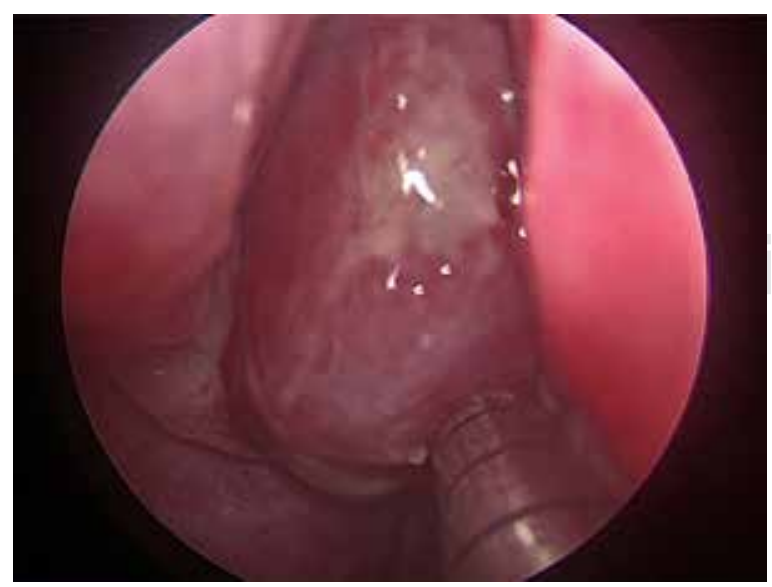

Figura 1: Imagen endoscópica de la tumoración dependiente del cornete medio derecho.
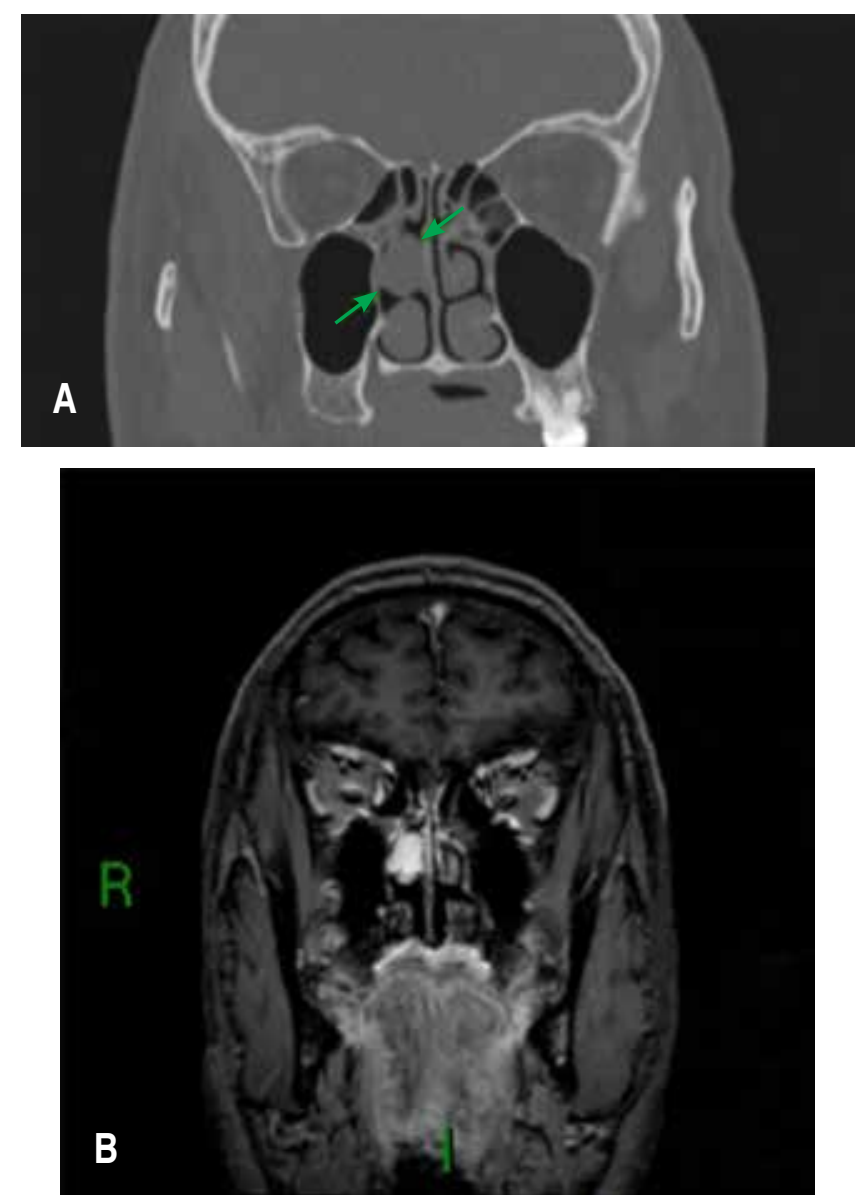

Figura 2: Imágenes de la tumoración por tomografía computarizada (A) y resonancia magnética (B). Lesión observada en tomografía computarizada en imagen $A$ de características redondeadas y bien definidas, en imagen $B$ se observa reforzamiento de la imagen en la resonancia magnética tras la aplicación de gadolinio.

\section{INFORME DE CASO}

Presentamos el caso de un paciente masculino de 43 años, sin antecedentes médicos previos. Gozaba de buena salud y es de un estrato socioeconómico medio-alto. No tiene antecedentes heredofamiliares de importancia.

Acudió a nuestra consulta tras referir dos cuadros de epistaxis que ameritaron manejo médico, requiriendo la colocación de un taponamiento nasal anterior, sin embargo, no cedió completamente. En la revisión, el paciente presentaba signos vitales normales, sin datos de compromiso hemodinámico. Al momento de realizar la exploración con endoscopia nasal, se encontró una lesión de aspecto rojizo-azulado y friable dependiente del cornete medio derecho, identificándose como el sitio de sangrado (Figura 1). 
Se solicitó una tomografía computarizada de nariz y senos paranasales, encontrándose una lesión de márgenes bien definidos, dependiente del cornete medio derecho, sin erosión ósea. Se complementó con una resonancia magnética con gadolinio, la cual mostraba una imagen de bordes irregulares, bien limitada dependiente del cornete medio, de comportamiento hipointenso en $\mathrm{T} 1$, la cual reforzaba con gadolinio y en T2 se mostraba hiperintensa. Ésta se extendía dorsalmente al cornete superior (Figura 2).

Entre los diagnósticos diferenciales, se encuentra el hemangiopericitoma, el nasoangiofibroma juvenil, el papiloma nasal invertido, lesiones polipoideas y neurogliomas. Dado que todas estas lesiones presentan cierto patrón vascularizado, aunque varían en su presentación clínica y radiológica, deben ser consideradas por el clínico a cargo del paciente.

Una vez protocolizado el paciente, se planteó como tratamiento quirúrgico la resección completa de la lesión por cirugía endoscópica nasal, con previa angioembolización por el Departamento de Radiología Intervencionista (Figura 3). Se realizó la resección en quirófano bajo visión endoscópica apoyada por neuronavegación (Medtronic, Fusion, Jacksonville, USA) para no dejar tumoración residual y con ello una posible recurrencia (Figura 4).

Se utilizó el microdebridador para extirpar la lesión (previa toma de biopsia) y se realizó hemostasia con el

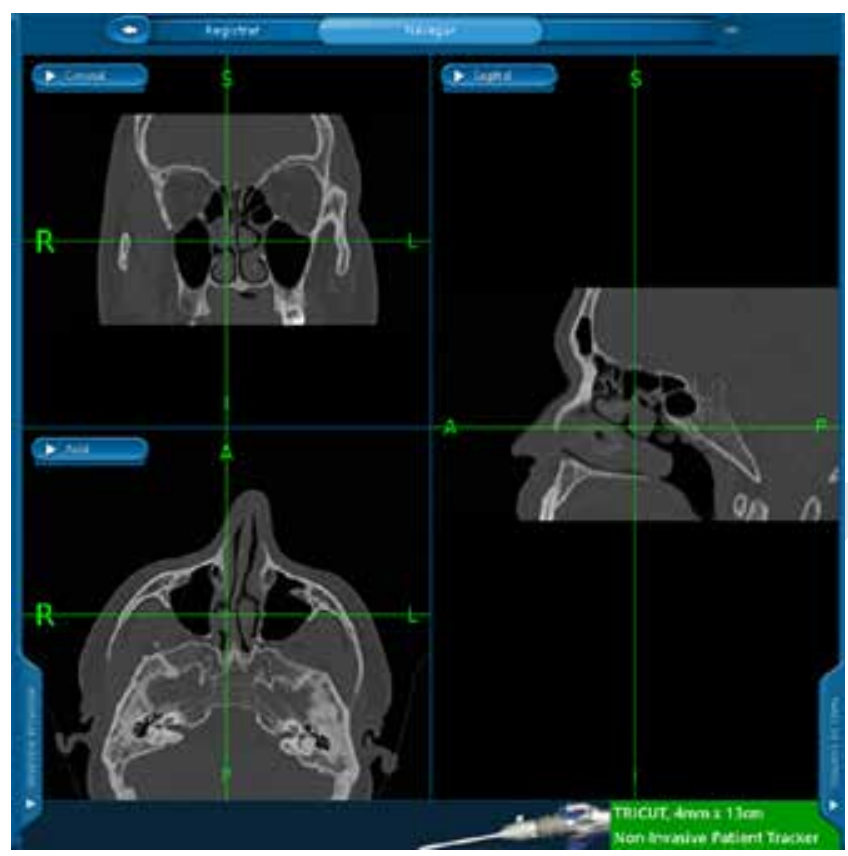

Figura 3: Localización de la lesión con neuronavegador. aspirador coagulador; gracias a la embolización previa, el sangrado no fue mayor. Se complementó la hemostasia colocando hemostático precursor de fibrinógeno de forma local. El paciente presentó adecuada evolución postoperatoria, sólo refiriendo cefalea relevante irradiada al ojo derecho, sin pérdida de la agudeza visual. Fue valorado por el oftalmólogo, quien sugirió inflamación del nervio infraorbitario; el dolor cedió a las 24 horas al colocar esteroide intramuscular y oral por cinco días. Por lo demás, su evolución fue satisfactoria.

El diagnóstico histopatológico definitivo fue un hemangioma capilar cavernoso (Figura 5). Seis meses posteriores a la resección, el paciente no presentó recurrencia.

El paciente fue intervenido satisfactoriamente gracias a una protocolización adecuada de la tumoración nasal. Los estudios de imagen permitieron delimitar adecuadamente la extensión de la lesión, la cual con ayuda de la endoscopia, el neuronavegador y la angioembolización permitieron su resección completa y sin recurrencia, además de prevenir un sangrado intenso en el transoperatorio. El trabajo multidisciplinario fue lo que generó el éxito de este caso en particular.

\section{DISCUSIÓN}

Existen diferentes reportes de caso de esta lesión en la cavidad nasal. En diferentes series, se han reportado lesiones en el septum, el cornete inferior y el piso nasal. Sin embargo, son aun más raras las lesiones localizadas en el cornete medio, por tanto, su consideración como un diagnóstico diferencial de epistaxis recurrente es relevante para el otorrinolaringólogo. ${ }^{4}$

La presentación clínica depende de la localización de la tumoración, pero en general es epistaxis recurrente, obstrucción nasal y la visualización de la masa, como se ha documentado en las diferentes series de casos, ${ }^{5-7}$ aunque puede ser variada dependiendo de sitios raros ocultos. El diagnóstico se ve apoyado por la exploración física, la endoscopia nasal y los estudios de imagen como resonancia magnética o tomografía computarizada con contraste.

El tratamiento está enfocado en la resección completa de la lesión, dado que no hacerlo presenta una recurrencia variable, la cual está reportada por diversos autores y oscila entre el 15 al 40\%. ${ }^{7-9}$

La escisión quirúrgica se puede realizar mediante visión directa, cirugía endoscópica nasal y asistida por una angioembolización, previa al procedimiento quirúrgico si se encuentra en un sitio del alto potencial de sangrado como el cornete medio. ${ }^{5,8,10-14}$ Asi- 


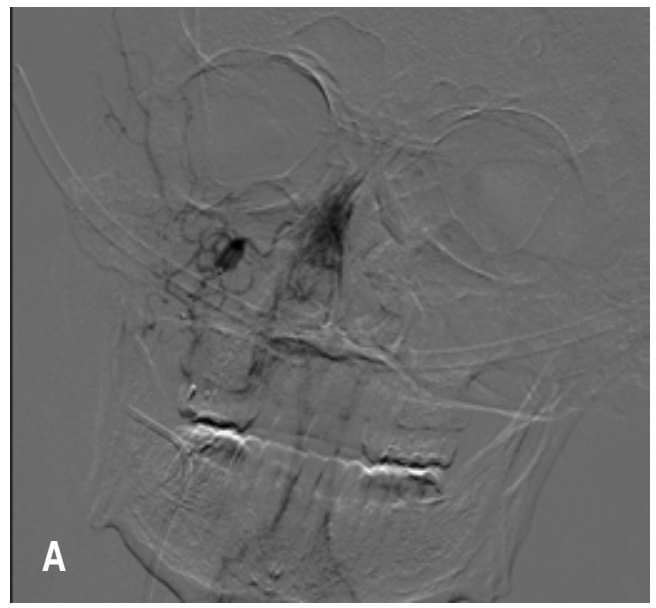

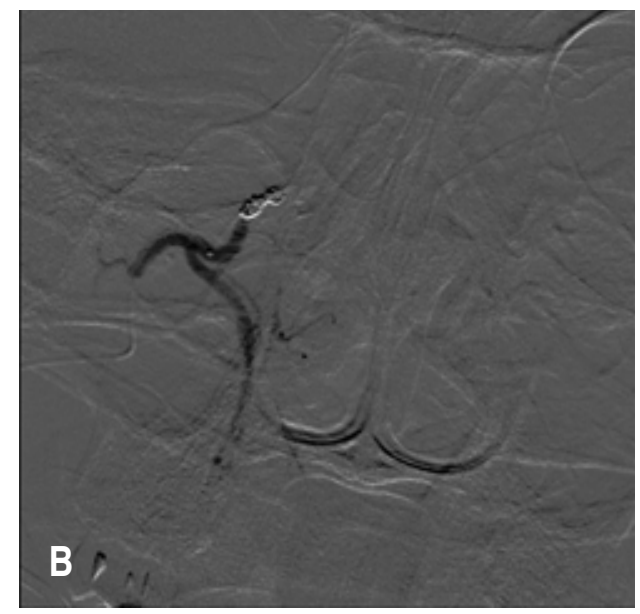

Figura 4:

Imágenes de la lesión previo tratamiento a angioembolización (A) y posterior a angioembolización (B).

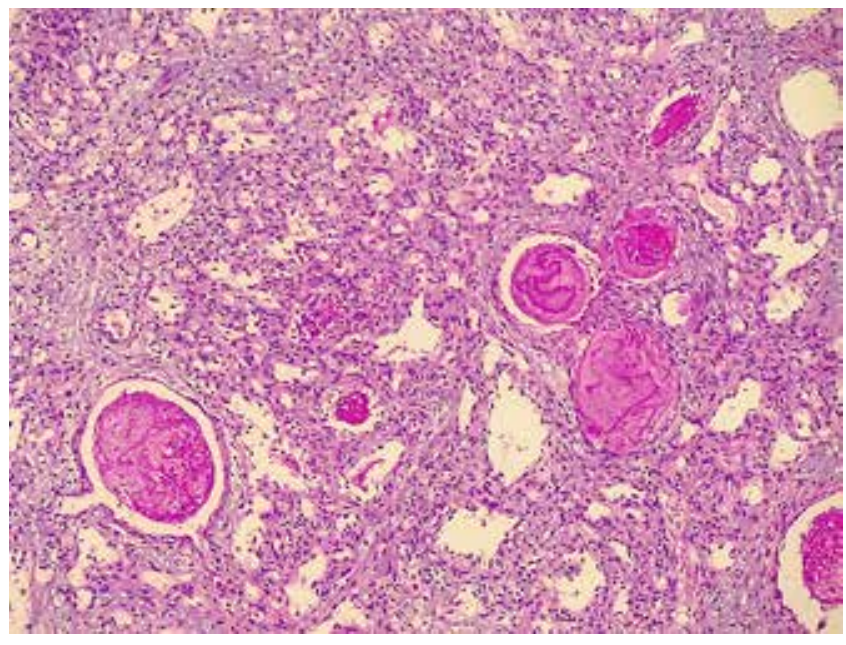

Figura 5: Imagen de histopatología. Hemangioma capilar lobular. Aspecto histopatológico. Tinción con hematoxilina/eosina. Vista a mediano aumento. Agregados lobulares de vasos sanguíneos separados por estroma fibroso.

mismo, se pueden utilizar abordajes combinados en localizaciones raras, como en la fosa pterigopalatina o el paladar. ${ }^{6,14,15}$ El pronóstico tras la resección completa es bueno y de carácter curativo.

Si bien los hemangiomas nasales ${ }^{2}$ son lesiones benignas, que en ubicaciones habituales no presentan una ruta diagnóstico-terapéutica compleja para el clínico, cuando se presentan en ubicaciones no habituales, es pertinente protocolizar adecuadamente al paciente. ${ }^{7}$ Sarkar y colaboradores ${ }^{4}$ presentaron el caso en el que el paciente presentaba anemia por una causa no detectable, hasta que se realizó el diagnóstico de un hemangioma en la porción posterior del cornete inferior y se resecó, lo que resolvió la causa.
Por su cuenta, Takaishi ${ }^{9}$ muestra en su estudio retrospectivo de 31 casos de hemangiomas, que cuando se presentan en una localización no habitual (septum nasal o cabeza del cornete inferior), el paciente puede llegar a presentar síntomas raros como alteraciones en la visión, y lo ejemplifica en un caso en el que el tumor se localizaba en el etmoides posterior. Otra localización extraña documentada es el caso reportado por Şahin, ${ }^{14}$ en el que el hemangioma se presentó en la fosa pterigopalatina, y el síntoma principal no fue epistaxis, sino sensación de plenitud facial y cefalea. Fue importante en este caso la utilización de resonancia magnética y tomografía computarizada, ${ }^{3}$ ya que la exploración endoscópica no presentaba ninguna anomalía. ${ }^{13}$

Las series de casos de esta patología son pequeñas dada su poca incidencia, ${ }^{6,15}$ pero la documentación de la misma ayuda a tener un mayor diagnóstico diferencial cuando el paciente presenta síntomas y signos que salen del patrón común de la presentación de la enfermedad.

\section{CONCLUSIÓN}

El caso representa una buena oportunidad para ejemplificar la presentación no habitual de una lesión benigna como lo es el hemangioma lobular capilar del cornete medio. La protocolización adecuada de la ruta diagnóstica y el tratamiento multidisciplinario fue clave para la adecuada resolución de la patología y para evitar la recurrencia de la misma.

Si bien quedan preguntas por resolver, como el dolor ocular que presentó el paciente, la limitada experiencia e información de estos casos en la literatura aporta un grado más de relevancia a la 
publicación de este caso clínico, el cual aporta un manejo ante el común diagnóstico de epistaxis para el otorrinolaringólogo.

\section{BIBLIOGRAFÍA}

1. Yau S. An update on epistaxis. Aust Fam Physician. 2015; 44 (9): 653-656.

2. Iwata N, Hattori K, Nakagawa T, Tsujimura T. Hemangioma of the nasal cavity: a clinicopathologic study. Auris Nasus Larynx. 2002; 29 (4): 335-339.

3. Kim JH, Park SW, Kim SC, Lim MK, Jang TY, Kim YJ et al. Computed tomography and magnetic resonance imaging findings of nasal cavity hemangiomas according to histological type. Korean J Radiol. 2015; 16 (3): 566-574.

4. Sarkar S, Roychoudhury A, Roychaudhuri BK. Intractable anemia: a case of bleeding nasal cavernous hemangioma. Indian J Otolaryngol Head Neck Surg. 2011; 63 (Suppl 1): 23-24.

5. Nouri H, Ziad T, Rochdi Y, Aderdour L, Raji A. Hémangiome capillaire caverneux du septum nasal. Rev Stomatol Chir Maxillofac. 2012; 113 (3): 176-178.

6. Testa D, Motta S, Massimilla EA, Tafuri D, Russo D, Russo A et al. Cavernous hemangioma of rhinopharynx: our experience and review of literature. Open Med (Wars). 2015; 10 (1): 523-528.

7. Tamaki A, Babajanian E, D’Anza B, Rodriguez K. Lobular capillary hemangiomas: Case report and review of literature of vascular lesions of the nasal cavity. Am J Otolaryngol. 2017; 38 (3): 363-366.

8. Özkiriş M, Aydin R, Seçkin S, Saydam L. A rare cause of pediatric nasal obstruction and epistaxis: Nasal septal mixed hemangioma. Int J Pediatr Otorhinolaryngol Extra. 2013; 8 (3): 104-107.

9. Takaishi S, Asaka D, Nakayama T, Iimura J, Matsuwaki Y, Hirooka S et al. Features of sinonasal hemangioma: A retrospective study of 31 cases. Auris Nasus Larynx. 2017; 44 (6): 719-723.

10. Masot-León B, Escudero-Nafs FJ, Ruiz de Azúa Ciria Y, NietoRamos H. Hemangioma cavernoso de huesos propios de la nariz: a propósito de un caso. Anales Sis San Navarra. 2018; 41 (3): 381-385.

11. Baki A. Nasal septal cavernous hemangioma. J Craniofac Surg. 2018; 29 (2): e135-e136.

12. Akiyama K, Karaki M, Osaki Y, Takeda J, Mori N. Intraosseous cavernous hemangioma of the middle turbinate. Auris Nasus Larynx. 2011; 38 (4): 516-518.

13. Al Washahi MK, Al Killidar A, Razek YA, Khamis T. Endonasal endoscopic complete excision of unilateral nasal hemangioma in a child: case report and technical note. Oman Med J. 2019; 34 (2): 156-159.

14. Şahin B, Sönmez S, Yılmazbayhan ED, Orhan KS. Cavernous hemangioma in unusual location: pterygopalatine fossa. Braz J Otorhinolaryngol. 2019; 85 (1): 121-124.

15. Kim JS, Kwon SH. Sinonasal hemangioma: diagnosis, treatment, and follow-up of 37 patients at a single center. J Oral Maxillofac Surg. 2017; 75 (8): 1775-1783. 